\title{
NEW SIMULTANEOUS APPROXIMATION FOR WAVE DIGITAL LATTICE FILTERS BASED ON THE ALTERNATIVE AND ITERATIVE GENERATION OF THE TWO BRANCH POLYNOMIALS
}

\section{Mohamed Yaseen, and}

Electrical Engineering Department, Faculty of Engineering, Assiut University, Assiut, Egypt, Email: M_yaseen3@yahoo.com

\section{Usama Sayed Mohammed}

Electrical Engineering Department, Faculty of Engineering, Assiut University, Assiut, Egypt, Email: Usama@aun.edu.eg

In this contribution, a new simultaneous amplitude and phase approximation for wave digital lattice structures is introduced. It is relying on the orientation of one of the two branch polynomials to adjust the amplitude, while the other is oriented to adjust the phase. The approximation process starts with proper initial settings for the two branch polynomials according to the given amplitude and phase specifications. Then, the two branch polynomials are generated alternatively. This means that during one polynomial is generated to approximate the amplitude or the phase, the other polynomial is fixed. By iterating this alternative procedure, the two polynomials and consequently the amplitude and phase converge to their optimal response. Interpolation method combined with the Remez-exchange algorithm is employed for this purpose. Finally, the filter structure is synthesized and the wave digital realization is reached.

KEYWORDS: wave digital filters, lossless lowpass lattice structures, simultaneous approximation.

\section{INTRODUCTION}

In literature [1-3], several design methods are available for wave digital lattice filters with only amplitude restrictions. For simultaneously specified amplitude and phase, some approximation methods have been established [4-6]. The common feature of these methods is the restriction of one of the two branch allpass functions to exhibit exact linear phase. The other allpass function is employed to control both the amplitude and phase. This needs to over-satisfy the amplitude or phase specifications. Consequently, the resulting filter structure is degree consuming. Recently, a simultaneous amplitude and phase approximation has been delivered [7] for wave digital lattice filters. This approximation avoids the restriction of one of the two allpass functions to have exact linear phase and guarantees equiripple amplitude and phase characteristics. It is relying on translating the amplitude and phase specifications into corresponding specifications for the difference and sum 
phase functions of two branch polynomials. Then, these difference and sum phase functions are approximated alternatively and iteratively until they converge into their optimal responses.

In this contribution, a new simultaneous amplitude and phase approximation is presented for wave digital lattice filters. It is relying on the orientation of one of the two branch polynomials to adjust the amplitude, while the other is oriented to adjust the phase. The approximation process starts with proper initial settings for the two branch polynomials according to the given amplitude and phase specifications. Then, the two polynomials are generated alternatively. This means that during one polynomial is generated to approximate the amplitude or the phase, the other one is fixed. By iterating this alternative procedure, the two polynomials and consequently the amplitude and phase converge into their optimal responses. Finally, the filter structure is synthesized and the wave digital realization is obtained by applying two-port parallel adaptors. The method is compared with the previous design methods from different points of view.

\section{THE THEORITICAL BASIS}

Let amplitude and linear phase specifications be given and it is required to approximate them simultaneously by a wave digital lattice structure. In this contribution we will consider typically the lowpass cases. The corresponding transmission function is formulated as:

$$
\mathrm{S}_{21}(\psi)=\frac{\mathrm{f}(\psi)}{\mathrm{g}(\psi)}
$$

where $\psi=\Sigma+\mathrm{j} \phi$ is the complex reference frequency variable. For lossless lowpass lattice structures [8], $\mathrm{f}(\psi)$ will be an even polynomial having degree $\mathrm{N}$ 1 , where $\mathrm{N}$ is the filter degree. On the other hand, $\mathrm{g}(\psi)$ is a strictly Hurwitz polynomial having degree N. For real reference frequencies, the transmission function is expressed as:

$$
\mathrm{S}_{21}(\mathrm{j} \phi)=\mathrm{A}(\phi) \mathrm{e}^{-\mathrm{jB}(\phi)}
$$

Consequently, $\mathrm{A}(\phi)$ and $-\mathrm{B}(\phi)$ are the amplitude and phase functions respectively. The polynomial $\mathrm{g}(\psi)$ can be decomposed as:

$$
\mathrm{g}(\psi)=\mathrm{g}_{1}(\psi) \mathrm{g}_{2}(\psi)
$$

where $g_{1}(\psi)$ and $g_{2}(\psi)$ are strictly Hurwitz polynomials having degrees $n_{1}$ and $\mathrm{n}_{2}$ respectively :

$$
\mathrm{n}_{1}=\frac{\mathrm{N}-1}{2} \quad \mathrm{n}_{2}=\frac{\mathrm{N}+1}{2}
$$

Now let the phase functions exhibited by $\mathrm{g}_{1}(\mathrm{j} \phi)$ and $\mathrm{g}_{2}(\mathrm{j} \phi)$ be $\theta_{1}(\phi)$ and $\theta_{2}(\phi)$ respectively. Mathematical processing yields: 


$$
\begin{aligned}
& \mathrm{A}(\phi)=\operatorname{Cos}\left[\theta_{2}(\phi)-\theta_{1}(\phi)\right] \\
& \mathrm{B}(\phi)=\theta_{2}(\phi)+\theta_{1}(\phi)
\end{aligned}
$$

Inversely,

$$
\begin{aligned}
& \theta_{2}(\phi)=\frac{B(\phi)+\operatorname{Cos}^{-1}[A(\phi)]}{2} \\
& \theta_{1}(\phi)=\frac{B(\phi)-\operatorname{Cos}^{-1}[A(\phi)]}{2}
\end{aligned}
$$

Consequently, Equations $(5,6)$ express the amplitude and phase functions $\mathrm{A}(\phi)$ and $\mathrm{B}(\phi)$ in terms of the two branch polynomials phase functions $\theta_{1}(\phi)$ and $\theta_{2}(\phi)$. On the other hand, Equations $(7,8)$ express the two branch polynomials phase functions $\theta_{1}(\phi)$ and $\theta_{2}(\phi)$ in terms of the amplitude and phase functions $\mathrm{A}(\phi)$ and $\mathrm{B}(\phi)$.

The approximation is relying on the orientation of $\mathrm{g}_{2}(\psi)$ to adjust the amplitude , while $\mathrm{g}_{1}(\psi)$ is oriented to adjust the phase. The approximation process starts with initial proper settings for $\mathrm{g}_{2}(\psi)$ and $\mathrm{g}_{1}(\psi)$ according to the given amplitude and phase specifications. Then, the two polynomials are generated alternatively. This means that during the polynomial $\mathrm{g}_{2}(\psi)$ is generated to approximate the amplitude , the polynomial $\mathrm{g}_{1}(\psi)$ is fixed.. Inversely, during the polynomial $\mathrm{g}_{1}(\psi)$ is generated to approximate the phase, the polynomial $\mathrm{g}_{2}(\psi)$ is fixed. By iterating this iterative procedure, the two polynomials and accordingly the amplitude and phase converge into their optimal response. Finally, the filter structure is synthesized and the wave digital realization is obtained.

\section{THE APPROXIMATION PROCEDURE}

The approximation procedure can be summarized in steps as follows:

1- Set initial value for the filter degree N. Consequently the degrees $n 1$ and $n 2$ can be determined according to Eq. (4). The initial value of $\mathrm{N}$ can be chosen to be equal to the degree of a lattice structure satisfying the amplitude specifications only [1].

2- An initial settings for $\mathrm{g}_{1}(\psi)$ and $\mathrm{g}_{2}(\psi)$ is carried out as follows:

At a set of digital frequency points ( $\mathrm{n}_{1}$ points) get the amplitude and phase specifications. Translate these specifications into corresponding phase specifications for $\theta_{1}$ and $\theta_{2}$ by applying Equations $(7,8)$. Then, $\mathrm{g}_{1}(\psi)$ can be generated. The additional phase specification value for $\mathrm{g}_{2}(\psi)$ (which lies in the stopband) is obtained from the generated $\mathrm{g}_{1}(\psi)$ and the corresponding amplitude specification value according to Eq. (5). Then, $\mathrm{g}_{2}(\psi)$ can be 
generated. The initial selected frequency points can be distributed over the passband and stopband such that they appear in each band in an equidistant arrangement with the band edges be fixed points. This has been detected to be sufficient for the convergence process. Note that the digital interpolation digital frequencies are reflected into the reference domain by applying the bilinear transformation [9]:

$$
\Omega=\frac{z-1}{z+1} \quad, z=e^{p T} \quad, \phi=\tan (\omega T / 2)
$$

where $p=\sigma+j \omega$ is the complex frequency variable in the digital domain and $T$ is the sampling period.

3- Now, the polynomial $\mathrm{g}_{1}(\psi)$ is fixed. The polynomial $\mathrm{g}_{2}(\psi)$ is newly generated to optimize the amplitude response. This is achieved by applying the Remez-exchange algorithm [10] to change the set of interpolation frequencies for $\mathrm{g}_{2}(\psi)$ according to the amplitude response and by applying Eq. (5). .

4- Now, with the amplitude specifications become satisfied, the polynomial $\mathrm{g}_{2}(\psi)$ is fixed. The polynomial $\mathrm{g}_{1}(\psi)$ is newly generated to optimize the phase response. This is achieved by applying the Remez-exchange algorithm to change the set of interpolation frequencies for $\mathrm{g}_{1}(\psi)$ according to the phase response and by applying Eq. (6).

The generation of $\mathrm{g} 1(\psi)$ and $\mathrm{g}_{2}(\psi)$ can be carried out by solving a set of linear equations to determine the coefficients. However, a recursive procedure which was delivered by Henk [11] is applied. According to this procedure, the phase function is specified by its values and/or higher derivatives at a set of frequencies. Note that a $\mathrm{k} \pi$ deviation from the specified phase value is possible, where $\mathrm{k}$ is integer. The polynomial is generated by recurrence formulae whose coefficients are calculated by a recursive algorithm. Let the number of the prescribed derivatives be $\mathrm{m}_{\mathbf{i}}$ at a specified reference frequency $\phi_{\mathbf{i}}$, where $\mathrm{O} \leq \mathrm{i} \leq \mathrm{r}$ and $\phi_{\mathrm{O}}=\mathrm{O}$. Without counting the zero-phase requirements at the origin, the number of the phase constraints is $n=r+\sum_{0}^{r} m_{i}$. A secondary set of frequencies $\mathrm{v}_{\mathrm{S}}$ is introduced with $0 \leq \mathrm{s} \leq \mathrm{n}$ comprising each frequency $\phi_{\mathbf{i}}\left(1+m_{i}\right)$ times. The ordering of the $\phi_{\mathbf{i}}$ frequencies in forming the set $v_{\mathrm{S}}$ is arbitrary except that $v_{0}=0$. Then, the polynomial which generally can be denoted by $Q_{n}(\psi)$ is generated according to the following recurrence formulae:

$$
\begin{gathered}
\mathrm{Q}_{0}(\psi)=1, \\
\mathrm{Q}_{1}(\psi)=\alpha_{0}+\psi \\
\mathrm{Q}_{\mathrm{k}+1}(\psi)=\alpha_{\mathrm{k}} \mathrm{Q}_{\mathrm{k}}(\psi)+\left(\psi^{2}+v_{\mathrm{k}}^{2}\right) \mathrm{Q}_{\mathrm{k}-1}(\psi), \mathrm{k} \geq 1
\end{gathered}
$$


where:

$$
\begin{gathered}
\alpha_{k}=\frac{X_{k}-1\left(j v_{k}+1\right)}{X_{k}\left(j v_{k}+1\right)}, k \geq 0, \\
X_{k}(\psi)=\frac{-\alpha_{k}-1 X_{k}-1(\psi)+X_{k}-2(\psi)}{\psi^{2}+v_{k}^{2}}, k \geq 1
\end{gathered}
$$

with the following initial conditions :

$$
\begin{aligned}
& \mathrm{X}_{-1}(\psi)=\mathrm{B}(\psi), \\
& \mathrm{X}_{0}(\psi)=\mathrm{A}(\psi) / \psi
\end{aligned}
$$

The functions $\mathrm{A}(\psi)$ and $\mathrm{B}(\psi)$ are related to the polynomial $\mathrm{Q}_{\mathrm{n}}(\psi)$ according to :

$$
\frac{\operatorname{Odd}\left(Q_{\mathbf{n}}(\psi)\right)}{\operatorname{Even}\left(Q_{n}(\psi)\right)}=\frac{A(\psi)}{B(\psi)}+\xi
$$

where $\xi$ is the error function:

$$
\xi=\frac{(-1)^{\mathrm{n}+1} \mathrm{X}_{\mathrm{n}}(\psi) \psi}{\mathrm{B}(\psi) \operatorname{Even}\left(\mathrm{Q}_{\mathrm{n}}(\psi)\right)} \prod_{1}^{\mathrm{n}}\left(\psi^{2}+v_{\mathrm{S}}^{2}\right)
$$

5- The steps $(3,4)$ are iterated alternatively until both the amplitude and phase specifications become satisfied. If the degree is not sufficient, it is increased by two and we return into step (2).

6- From the resulting two polynomials $g_{1}(\psi)$ and $g_{2}(\psi)$, synthesize the filter as follows:

\section{THE SYNTHESIS PROBLEM}

From the resulting two branch polynomials, the resulting two branch allpass functions are formulated:

$$
\mathrm{S}_{1}(\psi)=-\frac{\mathrm{g}_{1}(-\psi)}{\mathrm{g}_{1}(\psi)} \quad \mathrm{S}_{2}(\psi)=\frac{\mathrm{g}_{2}(-\psi)}{\mathrm{g}_{2}(\psi)}
$$

These two allpass functions are factorized into sections of first and second orders. A section of first order is formulated as:

$$
S_{i}(\psi)=\frac{-\psi+\beta_{i}}{\psi+\beta_{i}}
$$

The wave digital realization of this section results in a two-port parallel adaptor with coefficient value [1]: 


$$
\gamma_{\mathrm{i}}=\frac{1-\beta_{\mathrm{i}}}{1+\beta_{\mathrm{i}}}
$$

A section of second order is formulated as:

$$
S_{j}(\psi)=\frac{\psi^{2}-\alpha_{j} \psi+\beta_{j}}{\psi^{2}+\alpha_{j} \psi+\beta_{j}}
$$

The wave digital realization of this section results in a pair of two-port parallel adaptors with coefficient values [1]:

$$
\gamma_{2 j-1}=\frac{\alpha_{j}-\beta_{j}-1}{\alpha_{j}+\beta_{j}+1} \quad \gamma_{2 j}=\frac{1-\beta_{j}}{1+\beta_{j}}
$$

\section{DESIGN EXAMPLE}

The presented approximation method will now be applied through a design example. Suppose we are given the following amplitude and phase specifications:

- The passband is extending from 0 to $4.5 \mathrm{kHz}$ with maximum allowed loss $=0.01 \mathrm{~dB}$.

- The stopband is extending from 6.5 to $9.5 \mathrm{kHz}$ with minimum allowed loss $=30 \mathrm{~dB}$.

- The passband phase is required to be linear with allowed deviation from linearity $= \pm 0.1 \mathrm{rad}$.

- The sampling frequency $=19 \mathrm{kHz}$.

- The initial settings for the two polynomials $g_{1}(\psi)$ and $g_{2}(\psi)$ are obtained from the following interpolation values for $\mathrm{A}$ and $\mathrm{B}$ :

\begin{tabular}{||c|c|c||}
\hline \hline $\begin{array}{c}\text { FREQ. } \\
\text { KHZ }\end{array}$ & $\operatorname{Cos}^{-1}(\mathbf{A})$ & $\begin{array}{c}\text { B DEVIATION FROM } \\
\text { LINEARITY RAD. }\end{array}$ \\
\hline 1.5 & 0.04797559 & 0.1 \\
3 & -0.04797559 & -0.1 \\
4.5 & 0.04797559 & 0.1 \\
6.5 & 1.539168 & 1.9 \\
8 & 1.602424 & - \\
\hline
\end{tabular}

Then, by applying Equations $(7,8)$, the corresponding phase specifications for the two polynomials $g_{1}(\psi)$ and $g_{2}(\psi)$ are computed and the initial settings for the two polynomials are determined as: 


$$
\begin{aligned}
\mathrm{g}_{1}(\psi) & =\psi^{4}+3.520103 \psi^{3} 4.757282 \psi^{2}+3.367241 \psi+0.7916716 \\
\mathrm{~g}_{2}(\psi) & =\psi^{5}+3.308646 \psi^{4}+5.4812870 \psi^{3}+5.597234 \psi^{2}+4.076206 \psi \\
& +0.8669953
\end{aligned}
$$

Then, by following the approximation procedure according to steps (3-5), the final interpolation values for the two polynomials reached after 8 iterations are:

\begin{tabular}{||c|c||}
\hline FREQ. KHZ & $\boldsymbol{\theta}_{\mathbf{1}}$ RAD. \\
\hline 1.1875 & 0.80827 \\
3.55775 & 2.325049 \\
4.5 & 3.002257 \\
6.5 & 4.479437 \\
\hline
\end{tabular}

\begin{tabular}{||c|c||}
\hline FREQ. KHZ & $\boldsymbol{\theta}_{\mathbf{2}}$ RAD. \\
\hline 1.32525 & 0.946291 \\
3.78575 & 2.434206 \\
4.5 & 3.050233 \\
6.5 & 6.018604 \\
7.8755 & 6.941248 \\
\hline
\end{tabular}

These interpolation values result in the following final settings for the two polynomials:

$$
\begin{aligned}
\mathrm{g}_{1}(\psi)= & \psi^{4}+3.490021 \psi^{3}+4.748461 \psi^{2}+3.3401510 \psi+0.7946849 \\
\mathrm{~g}_{2}(\psi)= & \psi^{5}+3.309255 \psi^{4}+5.44198 \psi^{3}+5.84473 \psi^{2}+4.061367 \psi \text { Accordingly, } \\
& +0.890694
\end{aligned}
$$

the resulting loss response is shown in Figs. $(1,2)$. The resulting phase response is shown in Fig.(3). The phase deviation from linearity is shown in Fig.(4).

To obtain the wave digital realization, the two polynomials are factorized into first and second order sections:

$$
\begin{gathered}
\mathrm{g}_{1}(\psi)=(\psi+0.422223)(\psi+1.678601)\left(\psi^{2}+1.389198 \psi+1.121259\right) \\
\mathrm{g}_{2}(\psi)=(\psi+0.34749)\left(\psi^{2}+2.489772 \psi+1.858311\right) \\
\left(\psi^{2}+0.471994 \psi+1.379331\right)
\end{gathered}
$$




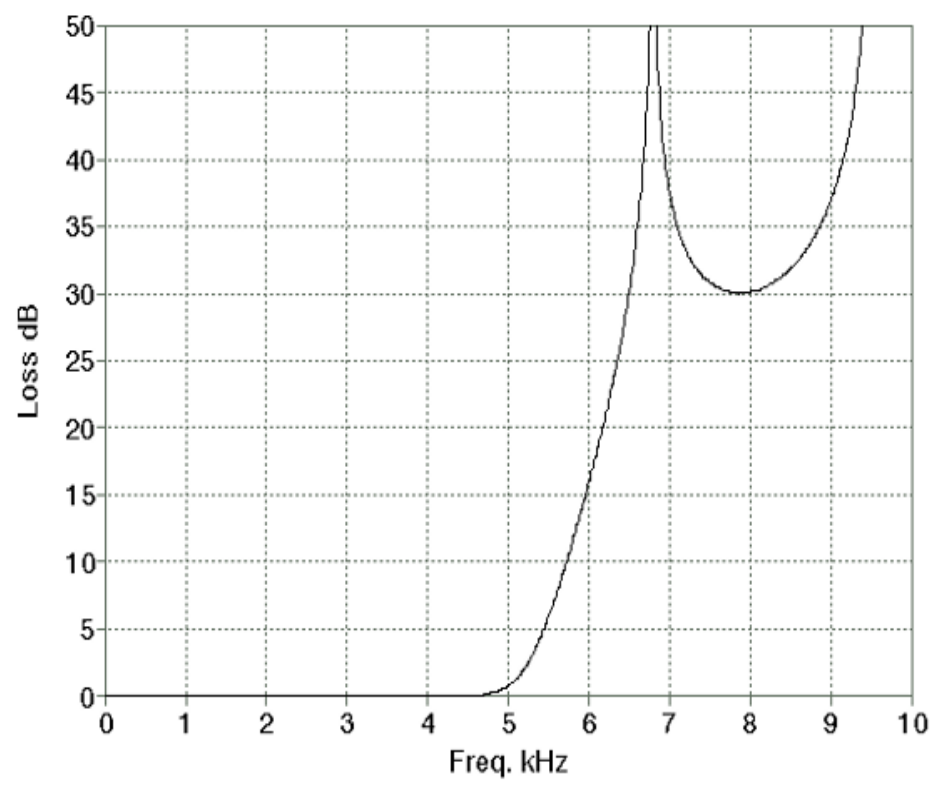

Fig. 1 The resulting loss response

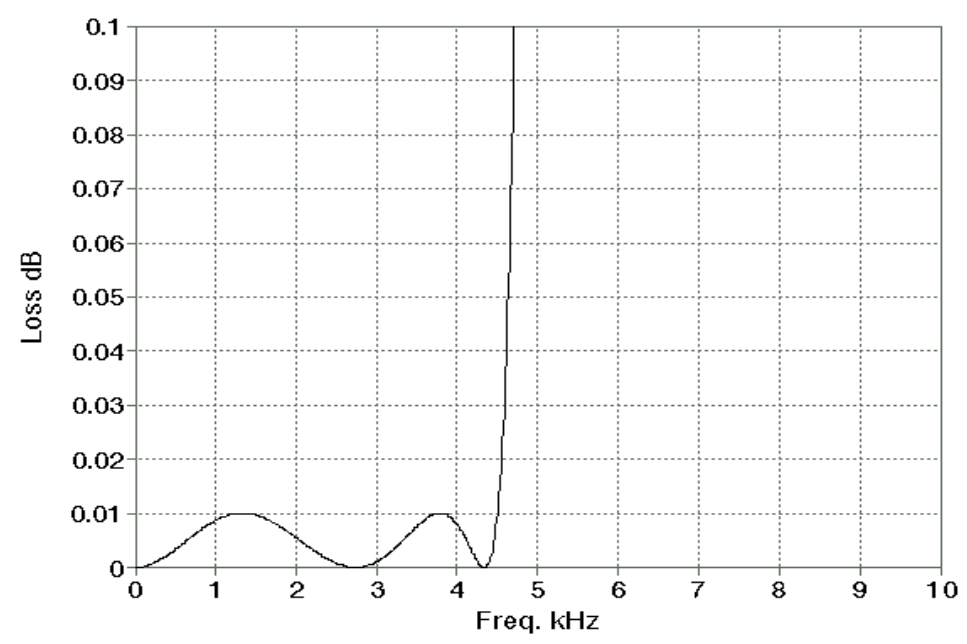

Fig. 2 The passband loss 


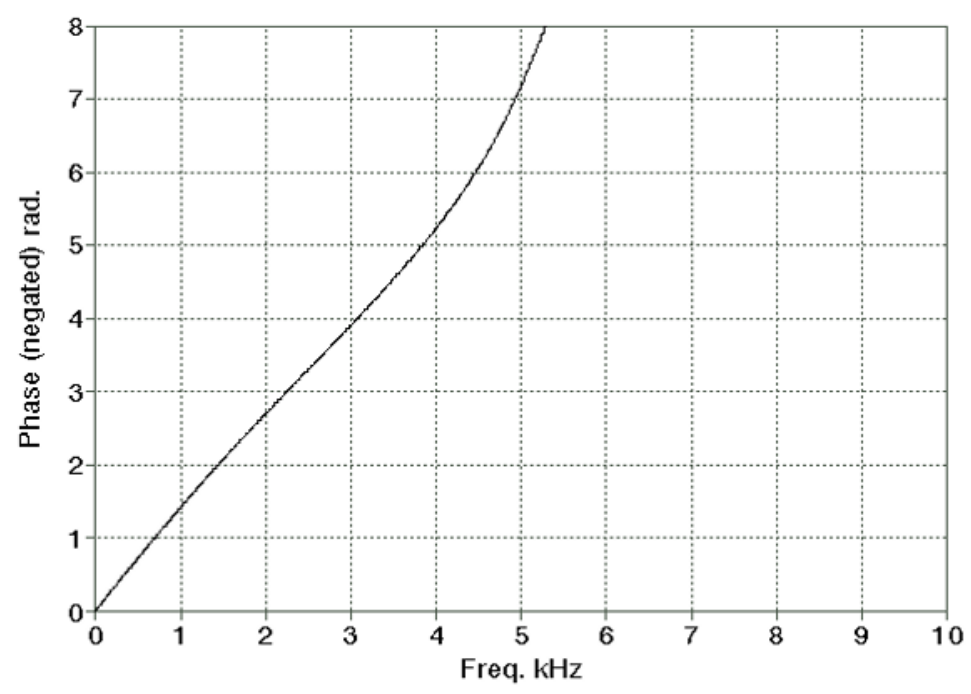

Fig. 3 The resulting phase response

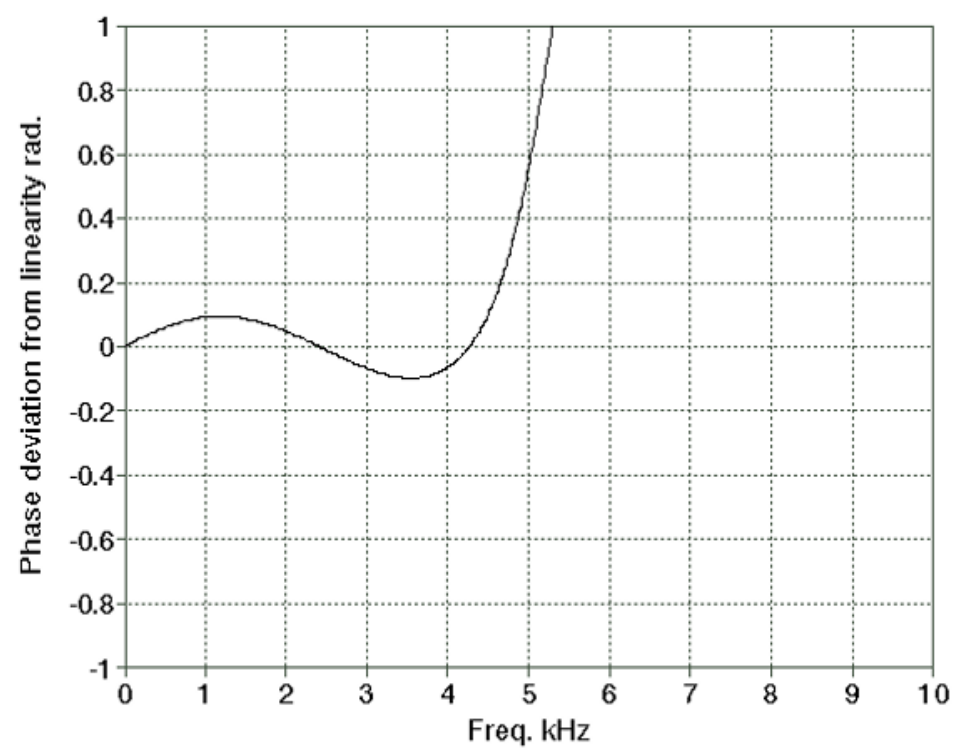

Fig. 4 The phase deviation from linearity

Accordingly, by applying Equations (15-18), the wave digital realization will be as shown in Fig.(5), with the following adaptor coefficient values:

$$
\begin{aligned}
& \gamma_{1}=0.4062493 \\
& \gamma_{3}=-0.2085372 \\
& \gamma_{5}=0.4842410 \\
& \gamma_{7}=-0.3002862 \\
& \gamma_{9}=-0.1594276
\end{aligned}
$$

$$
\begin{aligned}
& \gamma_{2}=-0.2533416 \\
& \gamma_{4}=-0.05716358 \\
& \gamma_{6}=-0.06891054 \\
& \gamma_{8}=-0.6689301
\end{aligned}
$$




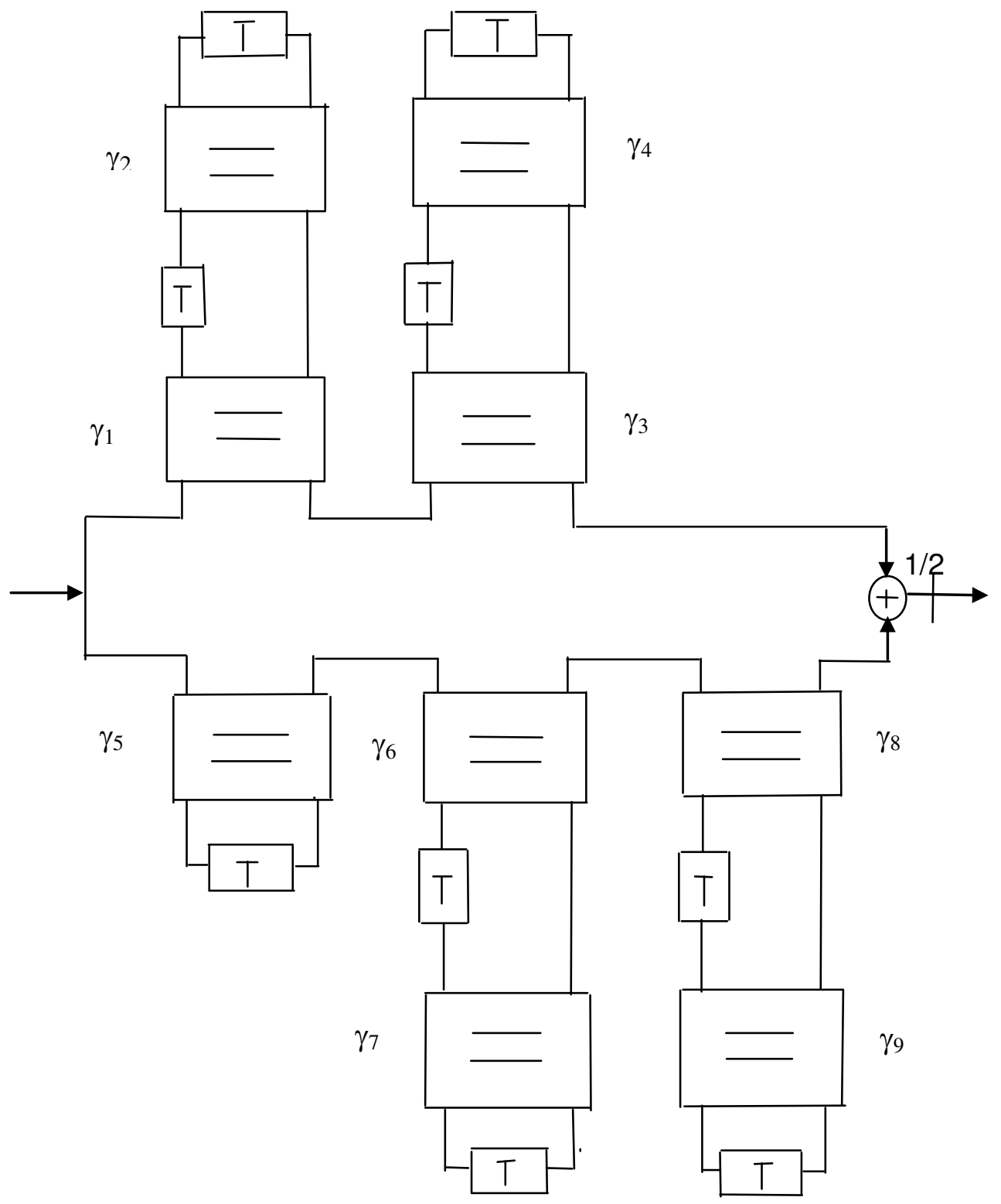

Fig.5. The wave digital realization.

\section{CONCLUSIONS}

Now, we will compare the delivered method with previous approximation methods [4-7]

1- The methods of references [4-6] are relying on the restriction of one of the two branch polynomials to exhibit exact linear phase. The other polynomial is employed to control both the amplitude and phase. Generally, this needs to 
over-satisfy the amplitude or phase specifications which results in noneconomic degrees. For the specifications considered in the example given above, the method of references [5-6] offers solution of degree 11.

2- The method of reference [7] is relying on the alternative and iterative approximation of the difference and sum phase functions of the two branch polynomials. In the phase of approximating the difference phase function, the sum phase function is fixed and the amplitude is adjusted. In the phase of approximating the sum phase function, the difference phase function is fixed and the phase is adjusted. In each phase, the two branch polynomials are newly generated. For the specifications considered in the example given above, this method [7] offers a solution of degree 9.

3 - The delivered method in this contribution is relying on the alternative and iterative approximation of the two branch polynomials $\mathrm{g}_{1}(\psi)$ and $\mathrm{g}_{2}(\psi)$. In the phase of approximating $\mathrm{g}_{2}(\psi)$, the polynomial $\mathrm{g}_{1}(\psi)$ is fixed and the amplitude is adjusted. In the phase of approximating $g_{1}(\psi)$, the polynomial $g_{2}(\psi)$ is fixed and the phase is adjusted.

\section{REFERENCES}

1. Gazsi,"Explicit Formulas for Lattice Wave Digital Filters", IEEE Trans. on Circuits and Systems, vol. CAS-32, pp.68-88, Jan. 1985.

2. M. Yaseen and T. Henk, "Synthesis Methods for Wave Digital Filtrs Exhibiting Arbitrary Amplitude Characteristics", Proc. of the 6-th European Conference of Signal Processing (EUSIPCO'92) vol. II, pp. 961-964, Brussels, Belgium, August 24-27, 1992.

3. Fettweis,"Wave Digital Filters, Theory and Practice", Proc. IEEE, vol. 74, pp. 270-327, Feb. 1986.

4. Kunold,"Linear Phase Realization of Wave Digital Filters", Proc. IEEE ISCASSP-88, IEEE, NewYork, 1988, pp. 1455-1458.

5. M. Abo-Zahhad, M. Yaseen and T. Henk, " Design of Lattice Wave Digital Filters with Prescribed Loss and Phase Specifications", Proc. of the European conference of Circuit Theory and Design (ECCTD'95), vol. 2, pp. 761-764, Istanbul, Turkey, Aug. 27-31, 1995.

6. M. Abo-Zahhad, M. Yaseen and T. Henk, "Arbitrary Amplitude and Linear Phase Approximations of Non-Prototype Ladder and Lattice Wave Digital Filters", the International Journal of Circuit Theory and Applications, vol. 24, no. 6, pp. 605- 620, Nov.-Dec. , 1996.

7. M. Yaseen, "On the Simultaneous Amplitude and Phase Approximations of Wave Digital Lattice Filters", the I.J. of Circuit Theory and Applications, vol. 31, pp. 465-472, 2003.

8. V. Belevitch, Classical Network Theory, Holden-Day, 1968. 
9. A.V. Oppenheim and R.W. Schafer, Digital Signal Processing, PrenticeHall, 1975.

10. G. C. Temes and J. C. Bincham, "Iterative Chebyshev Approximation Technique for Network Synthesis", IEEE Trans. On Circuit Theory, vol. 14, no. 1, pp. 31-37, 1967.

11. T. Henk, "The generation of Arbitrary Phase Polynomials by Recurrence Formulae", International Journal of Circuit Theory and Applications, vol. 9, pp. 461-478, 1981.

\section{"تقريب جديد متزامن للمرشحات الرقميه الموجيه ذات الهياكل الثبكيه أعتمادا على التقريب المتبادل والمتكرر لكثيرات الحدود الفرعيه"}

يقدم البحث طريقة فعالة لتصميم المرشحات الرقمية الموجية ذات الهياكل الثبكية وذات نطاق التمرير المنخفض Lowpass wave digital lattice filters بمواصفات سعة وطور خطى اختياريين . تعتمد الطريقة على الثقريب التبادلى Alternative و المتكرر Iterative لكل من متعددات الحدود الفرعيتين Two branch polynomials ـ يتم نوجيه كثيرة الحدود الأولى لضبط دالة السعة بينما يتم

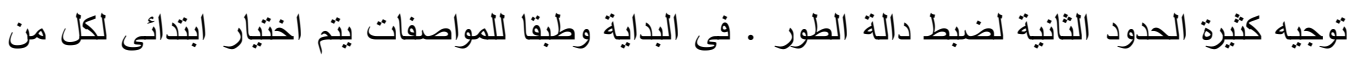

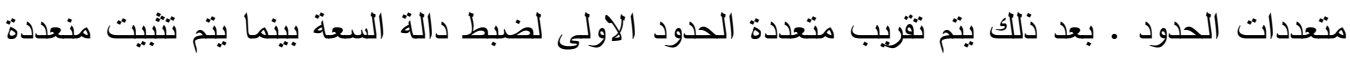

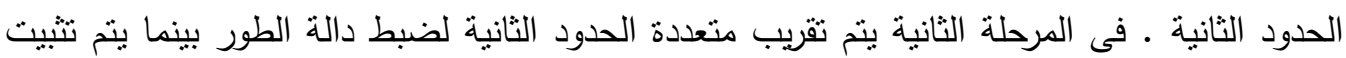

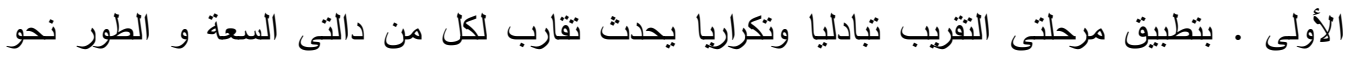

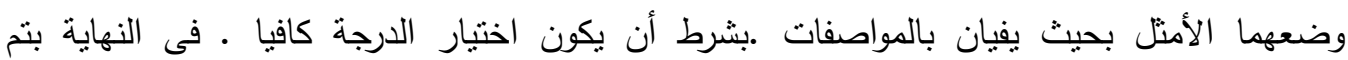
الحصول على كل من دالتى الامرار المطلق ويتم الحصول على الهيكل المرجعى والحصول على دائرة

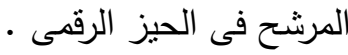

